\title{
Tip-of-the-tongue (TOT) states: retrieval, behavior, and experience
}

\author{
Bennett L. Schwartz • Janet Metcalfe
}

Published online: 4 January 2011

(C) Psychonomic Society, Inc. 2010

\begin{abstract}
The tip-of-the-tongue state (TOT) is the feeling that accompanies temporary inaccessibility of an item that a person is trying to retrieve. TOTs have been studied experimentally since the seminal work of Brown and McNeill (1966). TOTs are experiences that accompany some failed or slow retrievals, and they can result in changes in retrieval behavior itself, allowing us to study the interplay among experience, retrieval, and behavior. We often attribute the experience of the TOT to the unretrieved target, but TOTs are based on a variety of cues, heuristics, or sources of evidence, such as partial information, related information, and cue familiarity, that predict the likelihood of overcoming retrieval failure. We present a synthesis of the direct-access view, which accounts for retrieval failure, and the heuristic-metacognitive view, which accounts for the experience of the TOT. We offer several avenues for future research and applications of TOT theory and data.
\end{abstract}

Keywords Tip-of-the-tongue states · Metamemory ·

Metacognition $\cdot$ Retrieval $\cdot$ Lexical retrieval

As if waiting always for another word-

"at the tip of the tongue" we say, one thinks

of all the places tongues have been

and what they learned there, names.

—Robert Kelly (2009), "The Will of Achilles"

B. L. Schwartz ( $\square)$

Department of Psychology, Florida International University,

University Park,

Miami, FL 33199, USA

e-mail: bennett.schwartz@fiu.edu

J. Metcalfe

Columbia University,

New York, NY, USA

e-mail: jm348@columbia.edu
The poet Robert Kelly expressed in this poem an experience most of us have shared-that is, the strong feeling that we know something that we cannot recall at the moment, usually referred to as a tip-of-the-tongue state. We define tip-of-thetongue states (henceforth, TOTs) as the conscious feeling that accompanies or reflects upon the cognitive process of retrieval when an item that a person is trying to retrieve is temporarily inaccessible. The TOT entails two components: a basic level — the cognitive level — which is the act of trying to retrieve from memory, and a higher level - the metacognitive level-which is the commentary or reflection upon the cognitive level (Bacon, Schwartz, Paire-Ficout, \& Izaute, 2007). At the higher metacognitive level are conscious feelings: We are aware of this high-level reflection when we experience a TOT. At the lower cognitive level is the attempt to retrieve a missing item from memory. That is, TOTs have content: We feel we are about to be able to recall the item, although we cannot do so right now. Indeed, a TOT may be thought of as a premonition of possible retrieval success, rather than an experience of retrieval failure. Our focus will be on the TOT as a metacognitive experience.

This two-part definition connects TOTs to fundamental issues in the study of human psychology-namely how experience, cognition, and behavior are related-dating back to the earliest concerns in psychology (Tulving, 1989; see Costall, 2006; Woodworth, 1931). Introspectionists were interested in the nature of consciousness, but foundered, not only on the replicability of their results, but also on the relation of these results to observable behavior (cf. Costall, 2006). TOTs are conscious experiences but, unlike those studied by the early introspectionists, have a behavioral correlate, namely the retrieval of specific target words. It is our contention that the importance of studying the TOT phenomenon rests in its status as a case study of human subjective experience and how it relates to cognition and behavior. 


\section{Problem}

TOTs play an important role in a number of areas within cognitive psychology, including theories of memory, language production, and metacognition. From the point of view of memory, the study of TOTs has contributed to our understanding of the effects of interference on recall (see Brown, 1991; Kornell \& Metcalfe, 2006; Schacter, 2001). From the point of view of language production, TOTs have alerted researchers to retrieval efforts that have stalled or failed, as well as providing insight into the structure of the lexicon (Gollan \& Brown, 2006; Harley \& Bown, 1998). From the metacognitive view, TOTs are feelings that arise when retrieval fails. The latter view distinguishes the feeling of the TOT from the retrieval failure itself. One of our goals of this review article is to synthesize these disparate perspectives.

From the metacognitive perspective, we consider it possible that the TOT and retrieval processes are dissociable (e.g., Metcalfe, Schwartz, \& Joaquim, 1993; Schwartz, 2010). Because TOTs are produced by metacognitive processes, it should be possible to find experimental variables that will affect them without affecting recall and variables that will affect recall without affecting TOTs. We will return to evidence bearing on these dissociations later. But considering TOTs as metacognition opens up a number of avenues of inquiry. We suggest that TOTs serve a monitoring function, alerting us to the possibility of remembering when retrieval apparently has failed (Schwartz, 2006). In this sense, TOTs enhance the functioning of the retrieval system rather than being an annoying glitch in this system.

From the metacognitive perspective, feeling states are of functional significance (see Schwartz, 1999; Tulving, 1989). Consider the following analogy between TOTs and problems with your car: You turn on the ignition and notice that your "low tire pressure" signal is activated. The light informs you that there is a problem, but the light is not the problem itself. The TOT experience is like the signal-it alerts you to a problem with your retrieval system but is not the problem itself. Crucial to the analogy is that the "low pressure" signal is not the same thing as a tire low on air. Moreover, the analogy also illustrates the concept of metacognitive control. Your awareness of a problem allows you to do something about it-you can bring the car to the shop when you see the warning light, and with retrieval failure, and you can continue searching and cuing yourself for the missing word when you feel the TOT. Finally, it is possible that there could be something wrong with your "low pressure" signal system; it may light up when there is no problem with your tires. The problem now is not the tires, but the monitoring system. This is analogous to an illusory TOT (Schwartz, Travis, Castro, \& Smith, 2000), in which the monitoring system signals retrieval failure when none has occurred.

This article is a progress report on the state of metacognitive research on TOTs. Since Brown and McNeill's (1966) first empirical study on TOTs, there has been a steady stream of research. More than 40 years later, we have a handle on why TOTs occur (their functional significance) and the mechanisms that underlie the TOT experience. Moreover, in recent years, neuroimaging studies have begun to reveal the brain mechanisms responsible for TOTs. Although the emerging story of TOTs has grown, consistent with the general pattern that we see for many metacognitive phenomena, much still remains to be learned about them. In this article, we review the problem (why some researchers choose to study TOTs), the impact (what TOTs tell us about the relation of subjective experience, cognition, and behavior), and the trajectory (where TOT research is going in the near future).

\section{TOTs as a case study of phenomenology}

We have chosen to study TOTs because they provide a case study that can help us understand the nature of phenomenological experience (henceforth, simply experience) and its relation to cognition and behavior. We present four arguments as to why TOTs are ideal for the purpose of studying metacognitive experience.

(1) Universality TOTs are universal experiences among people of all languages and cultures. In a survey of languages, Schwartz (1999) found that nearly $90 \%$ of the languages surveyed expressed the feeling of temporary inaccessibility using the same "tongue" metaphor as in English. Many of these languages are unrelated to Indo-European languages (e.g., Cheyenne, Hausa, Vietnamese) but use the same metaphor. Moreover, in a cross-cultural study conducted in Guatemala, Brennen, Vikan, and Dybdahl (2007) identified a Mayan language, Q'eqchi', that lacks a specific term for TOTs. They tested for TOTs in participants, who were not literate in Spanish and knew little spoken Spanish. However, when the Spanish term was described to them, they reported having experienced TOTs many times before in Q'eqchi'. Brennen et al. found rates of TOTs among the Q'eqchi' speakers comparable to those among speakers of Western languages. Although Brennen et al. tapped into a population different than the typical college student population, the TOTs reported by the Mayan speakers closely resembled those of English-speaking college students.

Other evidence points to the universal nature of TOTs (see Brown, 2011). They are experienced by 
monolinguals and bilinguals (see, e.g., Gollan \& Acenas, 2004), as well as by ASL speakers (Thompson, Emmorey, \& Gollan, 2005). TOTs are also experienced by synesthetes (Simner \& Ward, 2006). Across developmental trajectories, TOTs are experienced by children (Hanly \& Vandenberg, 2010), college-age students (Schwartz, 2006), and older adults (Brown \& Nix, 1996; Schwartz \& Frazier, 2005). They are also noticeable in a number of neurological conditions, including Alzheimer's disease, anomic aphasia, and temporal-lobe epilepsy (Brown, 2011).

(2) Frequency of experience Diary studies have shown that TOTs occur about once a week for younger adults and increase to about once a day for older adults (Dahlgren, 1998; Heine, Ober, \& Shenaut, 1999; see Brown, 2011). This contrasts TOTs with other experiences, such as déjà vu, which may only occur once or twice in a lifetime (Brown, 2004).

(3) Observability in lab TOTs are easily induced in the laboratory (Brown, 1991, 2011; Smith, 1994). In a half-hour experiment, a researcher may be able to generate numerous TOTs from a single participant, thereby allowing the researcher to examine the effects of a variety of experimental variables on the number, accuracy, and resolution of TOTs. Because of their ease of generation in the lab, TOTs can serve as a case study for examining the nature and effects of metacognitive experience.

(4) Referent in behavior Another feature of TOTs that recommends them as a case study of experience is that they are tied to a specific mental referent, namely the retrieval of a particular word. However obvious this fact is, it contrasts the TOT with other kinds of experiences. Déjà vu is a vague feeling that one has been somewhere before (Brown, 2003, 2004). Although Cleary, Ryals, and Nomi (2009) have been successful at inducing déjà vu experiences in the lab, it is still unclear what would make a déjà vu experience either accurate or illusory, because the familiarity that produces them is not as closely tied to an actual specific target as in TOTs. Sedikides, Wildschut, Arndt, and Routledge (2008) examined the experience of nostalgia. Like déjà vu, nostalgia is an experience associated with memory, but in many cases it may lack a referent other than some earlier time in the person's life. A TOT has a referent, the target that the participant is trying to retrieve. For TOTs, accuracy is determined by examining if the person eventually can recall or recognize the target, and in general, TOTs tend to be accurate (Brown, 1991, 2011); that is, there is a positive correlation between TOTs and the likelihood of eventual recall (sometimes called resolution) and a positive correlation between TOTs and the likelihood of recognition.

To summarize, TOTs are an important metacognitive state because of their universality, the frequency of the experience in everyday life, their ease of observability in the lab, and because they have a referent in behavior. These characteristics make TOTs a good candidate for a case study in the scientific examination of human phenomenology, in particular the relation between subjective experience, cognition, and behavior. To be more specific, TOTs serve to aid memory function by alerting us to those apparent retrieval failures that are potentially resolvable.

\section{Impact}

We focus on the role that TOTs play in understanding the heuristic nature of metacognition and the role that the cues and clues play in producing TOTs. We also focus on the give and take between monitoring and control in metacognition and what TOTs can tell us about the control of retrieval. In both of these areas, TOT research has uncovered results that challenge how we think about the nature of retrieval and the nature of metacognition. In reviewing these topics, we will first need to review the differences between direct-access theories of TOTs and inferential theories, as well as to describe a synthesis of the two views.

\section{The direct-access view}

Early researchers on TOTs (Brown \& McNeill, 1966; James, 1890; Koriat \& Lieblich, 1974) assumed that TOTs were caused by unconscious access to the actual to-beretrieved target. In this view, TOTs occur when a person attempts to retrieve target words, but something blocks, interferes with, or prevents the retrieval of the target word. The target word has sufficient activation to trigger the TOT, but does not have sufficient activation to trigger recall (Brown \& McNeill, 1966; Perfect \& Hanley, 1992). This assumption still guides research on the phenomenon (Galdo-Alvarez, Lindin, \& Diaz, 2009; Hamberger \& Seidel, 2003; Lesk \& Womble, 2004). However, newer models postulate multiple processes of retrieval or multiple levels of representation (see Brown, 2011; Gollan \& Brown, 2006; Kornell \& Metcalfe, 2006). In all of these models, the goal is to use TOTs as markers of the interrupted or slow retrieval.

Research shows that, during TOTs, participants can sometimes retrieve partial information about the target 
itself and semantic information related to the target. The partial information includes phonological information, such as the starting sound and numbers of syllables (Koriat \& Lieblich, 1974). It can also include syntactical information such as the grammatical gender (Miozzo \& Caramazza, 1997). Bilinguals can sometimes access the translation equivalent in their other language (Gollan \& Acenas, 2004; Gollan \& Brown, 2006). In an interesting experiment, Hanley and Chapman (2008) demonstrated that participants could remember if actors and actresses were known by three names (e.g., Catherine Zeta Jones, Sarah Jessica Parker) rather than two (e.g., Gwyneth Paltrow, Cameron Diaz), even when they could not actually recall the names themselves. Finally, Simner and Ward (2006) found that synesthetes could recall the sensory characteristics associated with unretrieved TOT items. Thus, it is clear that, at least some of the time, participants do have access to partial information about the target when they are in a TOT state.

A number of models of lexical access focus on the difference between retrieval of semantic information, which occurs successfully during most TOTs, and the retrieval of complete phonological information, which is what fails, thereby leading to the conditions that cause the TOT (Gollan \& Brown, 2006). According to these models, retrieval occurs in two steps. First, a person retrieves meaning-based information (and perhaps syntactic information). Second, the phonological form is activated. A cue or question will prompt the retrieval of the semantic information. If that semantic information does not activate the appropriate phonological information, a TOT occurs. Gollan and Brown offer an up-to-date and complete account of this model.

The two-step view of TOTs leads to two hypotheses that were not present in the original Brown and McNeill (1966) formulation of the direct-access view. First, TOTs may stem from partially retrieved items rather than unretrieved (i.e., unconscious) items. The participant may recover much semantic and grammatical information about the target. Second, TOTs might indicate increased activation of targets rather than the failure to retrieve them (see Gollan \& Brown, 2006). Kornell and Metcalfe (2006) provided support for this contention. They showed that TOTs that people labeled as "blocked" TOTs had the same retrieval characteristics as "pure" TOTs. Blocked TOTs are those in which the person perceives that a retrieved answer is incorrect and is interfering with the retrieval of the correct answer. Pure TOTs are those in which no retrieval, correct or incorrect, is forthcoming. Kornell and Metcalfe argued that there was no evidence for the notion of interference accruing to the self-generated blockers that are often seen in TOT states. In Gollan and Brown's two-level model, TOTs result when the semantic form, or some close approximation to the semantic form, is retrieved, but the phonological form is not. As we shall see, this account of TOTs is compatible with, although not identical to, the heuristicmetacognitive account, which hypothesizes that retrieved semantic, syntactic, and phonological information combines with other related information to inform a metacognitive monitor that retrieval of the word is likely. What the metacognitive model adds to the Gollan-Brown model is the presence of a metacognitive monitor that produces a TOT feeling and regulates the flow of information. We will return to a proposed synthesis of the heuristic-metacognition model and the Gollan-Brown model shortly.

Some experimental research suggests that direct target access, even in a partial form as outlined above, may not be the only source of TOTs. Cleary (2006) examined the relation of TOTs to the familiarity of the questions, finding evidence for the proposal that factors other than just the retrieval of partial information about the target may contribute to TOTs. Cleary's results, therefore, mitigate against even a modified traditional direct-access view of TOTs. In her experiment, she presented participants with general-information questions. Unbeknownst to the participants, they had seen the answers to some of the questions in an earlier "unrelated" task. During the generalinformation task, participants did not have more TOTs for questions whose answers had been seen earlier than for questions whose answers had not been seen. Thus, priming the unrecalled targets did not increase the likelihood that those items would later be experienced as TOTs. Target priming did increase the likelihood that the person would judge that the answer to the unanswered question had been presented earlier. However, the fact that target priming did not affect TOTs runs counter to the view that target strength alone is the only possible source of TOTs.

Schwartz (2008) found evidence suggesting that something else was needed in addition to the modified directaccess view, in an examination of whether there was interference between TOTs and working memory. General-information questions were used to induce TOTs (e.g., "What is the last name of the composer who wrote Don Giovanni?"). After unsuccessful retrieval of a target, participants were presented with a series of digits and asked to keep those digits in mind (i.e., a digit span task) for half of the trials. For the other half of the trials, there was no concurrent memory load. Participants made TOT judgments for the general-information questions that they could not recall and then attempted to recognize the correct answer for those questions. After the participants made the TOT and recognition judgments, they were required to recall the digits. The results showed that relative to the control condition, there were fewer TOTs when participants were maintaining a digit load. However, recognition was not impaired by the working memory load. Thus, the digit span task affected TOTs rates (by lowering them), but did not 
affect the memorability of the target, as measured by recognition of the unrecalled items. That is, a factor other than the strength of the target was driving the TOTs, arguing against direct access as the sole source of TOT feelings.

The heuristic-metacognitive account

The heuristic-metacognitive account explains TOTs by focusing on the sources of information that people use to arrive at a TOT (see Dunlosky \& Metcalfe, 2009, for applications of this approach to other metacognitive judgments). When a person fails to recall a word immediately, metacognitive processes are activated, which are designed to inform the person of whether or not the word is potentially retrievable. The monitor does so by examining the amount of related and partial information recalled, the familiarity of the cue, and even the recent history of retrieving the particular word (Warriner \& Humphreys, 2008). That is, it is not subthreshold activation of the actual word alone that triggers the TOT, or even necessarily partial recall of the target, but a host of accessible cues and clues, which may include partial or whole activation of a bit of the target, or even of the whole target itself, if the person is not sure that the answer is correct (see Fig. 1).

Although it is tempting to think of the metacognitive monitor as being a kind of homunculus, it is not necessary to do so. In the CHARM model, for example, Metcalfe (1993) proposed a metacognitive monitor to explain feeling-of-knowing judgments that was a simple accumulator of feature matches and mismatches (see also Hazy, Frank, \& O'Reilly, 2007). The magnitude of the match mapped directly onto the judgments. In the present case, the metacognitive monitor need not be more than an accumulator of retrieved information from all sources that produces a TOT response at a certain criterion. When the amount of information accumulated reaches that predetermined criterion, it triggers a TOT state, which in turn controls the system to attempt to retrieve more information rather than quitting, as is shown in Fig. 1.

In some cases, the information that triggers the TOT need not include any part of the target at all, but may relate only to the question, not the answer. Thus, if a person is able to retrieve that an actress uses three names rather than two, this suggests to the metacognitive monitoring system that the person may also possess the actual name, albeit in a currently inaccessible state. In Koriat's (1993) theory, the metacognitive monitor counts the amount of information retrieved in order to derive a judgment. In Metcalfe's (1993) theory, the familiarity of the cue or question is paramount. In this context, familiarity means the ease of processing or fluency of processing the cue terms. If the question is familiar (e.g., "What was your great grandmother's name?"), we are more likely to experience a TOT than if the question is not familiar (e.g., "What is the capital of Kyrgyzstan?"). Like other heuristic judgments, these mechanisms are usually successful because most, but not all, cues and clues are correlated with the actual likelihood
Fig. 1 The heuristic-metacognition monitor weighs the evidence associated with a retrieval failure to determine whether a TOT is warranted

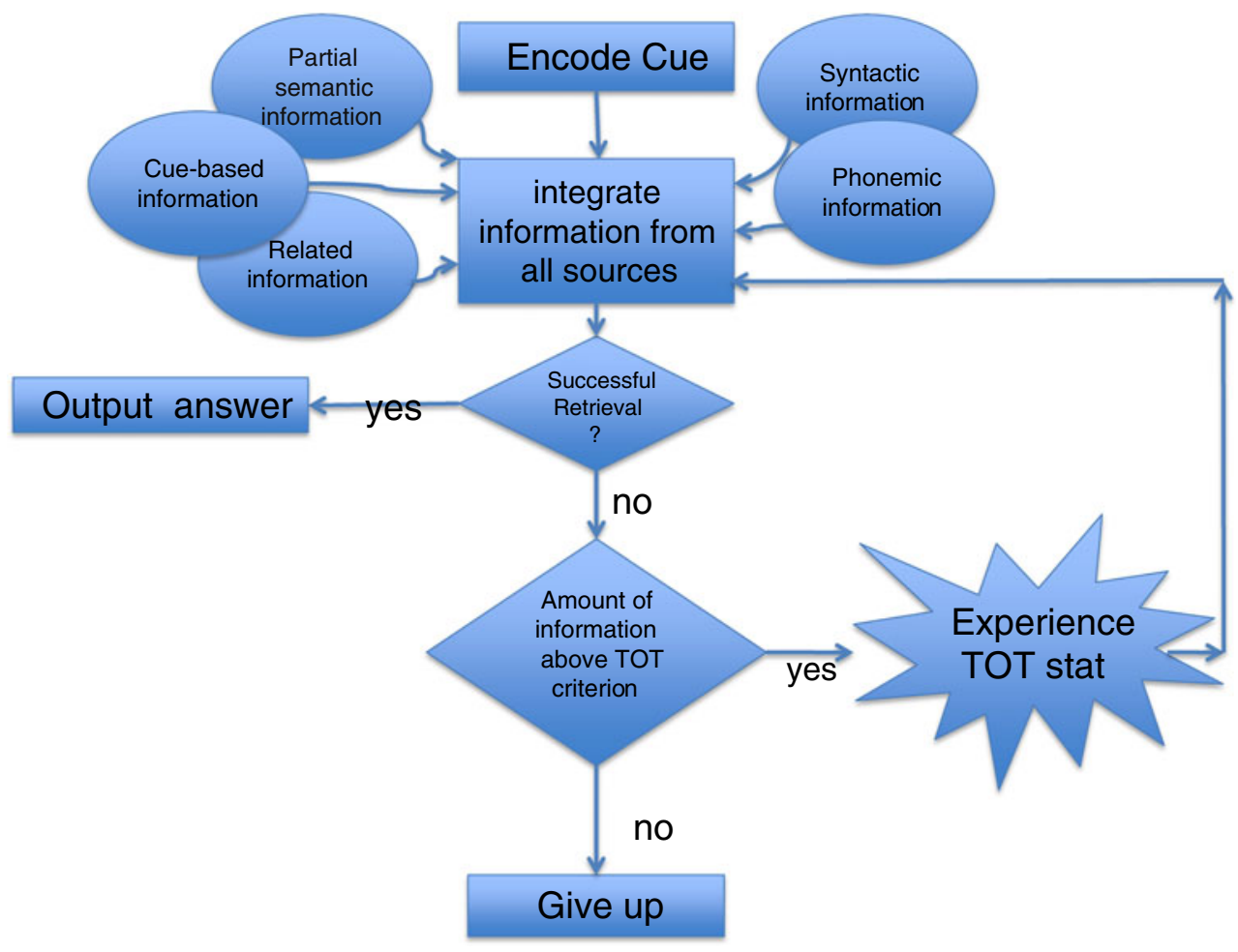


of maintaining the TOT target in memory (see Cleary, 2006; Metcalfe, 1993).

In Koriat's (1993) view, it is not the correctness of information that matters to the metacognitive monitor, but only the amount of information. Koriat (1993) claimed that we have no objective way to determine whether our retrieved information is correct. ${ }^{1}$ For example, when asked what is the capital of Australia, many non-Australian participants may experience a TOT, based on partial information, but this TOT may be directed incorrectly at the name "Sydney" rather than "Canberra." So, any partial or related information the person retrieves about Sydney will fuel the TOT, even though this answer is incorrect. In lexical-retrieval theory, these TOTs are often dismissed as negative TOTs and not further considered (Lesk \& Womble, 2004). However, for metacognitive views, the study of these TOTs is critical.

Behavioral evidence for the heuristic-metacognitive account

The earliest evidence in support of the idea that information not directly related to the target might contribute to the TOT, as proposed by the heuristic-metacognitive account, came from Koriat and Lieblich (1977), who reexamined their earlier TOT data (Koriat \& Lieblich, 1974) to determine what factors influenced participants' rates of TOTs. They identified that the cue or question contributed to the number of TOTs people reported when they were unable to recall the target. Participants indicated more TOTs for definitions that were long or redundant (e.g., "nimbus: a circle, or any indication of radiant light, around the head of divinities, saints, sovereigns in pictures, medals, etc.") than for definitions that were shorter and more concise (e.g., "numismatics: the science of coins"). That is, cues that contained more information were more likely to evoke a sense of familiarity than were shorter cues with less information. It is possible that better cues led to stronger target activation, but it is also possible to interpret these results in favor of the view that the familiarity of the cues

\footnotetext{
${ }^{1}$ In its strong form, Koriat's $(1993,1995)$ claim that people cannot distinguish between correct and incorrect retrieved information is incorrect. People can often tell if the information they produce is right or wrong, and they do not automatically think that everything they produce is necessarily correct. In Kornell and Metcalfe's (2006) experiments, for example, when people produced blockers-a frequent occurrence-they always knew that the blocker was incorrect. People's metaknowledge that the item that comes to mind is incorrect is routine with blockers. Even so, the essence of Koriat's argument may still be correct, in that even incorrectly retrieved information - and even incorrect information that the person knows full well is incorrect, as described above when the person is in a blocked TOT - could nevertheless inflate metacognitive feelings such as those experienced in the TOT state.
}

themselves produced the TOTs, consistent with the heuristicmetacognitive account (see Table 1).

More evidence for the heuristic-metacognitive view comes from Metcalfe et al. (1993), who compared the direct-access and heuristic-metacognitive theories. In their Experiment 3, they were interested in the role of cue familiarity in feeling-of-knowing judgments and TOTs. Participants studied word pairs such as "captain-carbon." In one condition, both the cue and the target were repeated (A-B, A-B) at time of study. In a second condition, the cue was repeated, but paired with an unrelated $\operatorname{target}(\mathrm{A}-\mathrm{B}, \mathrm{A}-$ D). This induces more fluent processing of the cue but should lead to interference in cued recall. In a third condition, neither the cue nor the target was repeated (AB, C-D). After the study session, participants were given an opportunity to recall the target word given the cue word. In accordance with the results from interference paradigms, the $\mathrm{A}-\mathrm{B}, \mathrm{A}-\mathrm{D}$ condition showed the lowest recall. Recall was highest in the $\mathrm{A}-\mathrm{B}, \mathrm{A}-\mathrm{B}$ condition. If participants could not recall the target, they made a TOT judgment. If direct access to the target caused TOTs, the expectation was that the A-B, A-B condition would produce more TOTs than other conditions because it contained the most strongly encoded items. Heuristic-metacognitive theory, which includes cue familiarity, predicted that TOTs would track the repetition of the cue. That is, the A-B, A-B and A-B, $\mathrm{A}-\mathrm{D}$ conditions should produce equal numbers of TOTs because the cue was repeated, and both should produce more TOTs than the A-B, C-D condition. Indeed, TOTs were highest in the two conditions in which the cue was repeated. In fact, the condition that produced the lowest recall $(\mathrm{A}-\mathrm{B}, \mathrm{A}-\mathrm{D})$ produced the highest number of TOTs. This suggested to Metcalfe et al. that participants were using cue familiarity as a heuristic to determine TOTs.

Schwartz and Smith (1997) found evidence to support the heuristic-metacognitive view of TOTs using a different paradigm. They asked participants to study the names, pictures, and biographical information of fictional animal species. Participants were instructed that each name referred to an animal and that the country was the habitat of that animal. For example, the participants saw "Yelkey-Panama," which indicated that the "yelkey" is an animal that lives in Panama. Of the animals for which drawings were provided, half were accompanied by information pertaining to the size and diet of the animal. This led to three encoding conditions: minimum information (just the name-country pair), medium information (the name-country pair and the line drawing), and maximum information (the name-country pair, the line drawing, and the additional biographical information). At test, participants were given the name of the country (e.g., Panama) and asked what animal came from that country. Recall of the target names (e.g., yelkey) was not significantly different across conditions. 
Table 1 Evidence for the heuristic-metacognition approach to TOTs

\begin{tabular}{lll}
\hline Study & $\begin{array}{l}\text { Behavioral } \\
\text { or Neural }\end{array}$ & Finding \\
\hline $\begin{array}{l}\text { Koriat and Lieblich (1977) } \\
\text { Metcalfe et al. (1993) }\end{array}$ & $\begin{array}{l}\text { Behavioral } \\
\text { Behavioral }\end{array}$ & $\begin{array}{l}\text { Longer definitions led to more TOTs } \\
\text { TOTs are caused by cue familiarity }\end{array}$ \\
Maril et al. (2005); Maril et al. (2001) & $\begin{array}{l}\text { Behavioral } \\
\text { TOTs are caused by related information }\end{array}$ & $\begin{array}{l}\text { TOTs were associated with activation in right } \\
\text { prefrontal cortex }\end{array}$ \\
Warriner and Humphreys (2008) & Behavioral & $\begin{array}{c}\text { History of retrieval failure led to more TOTs } \\
\text { Schwartz (2010) }\end{array}$ \\
Behavioral & $\begin{array}{c}\text { Emotional questions are more likely to induce } \\
\text { emotions than are nonemotional questions }\end{array}$ \\
Cleary et al. (2010) & Behavioral & $\begin{array}{c}\text { Items in TOT states were more likely judged to have } \\
\text { been studied in an earlier phase of the experiment }\end{array}$ \\
\hline
\end{tabular}

If participants used the amount of related information accessible as a mechanism to determine TOTs, there should have been more TOTs in the conditions that had more related information (see Koriat, 1993, 1995)—namely, in the medium and maximum information conditions. And the results showed that, indeed, there were more TOTs in the medium and maximum information conditions (19\%) than in the minimum information condition (11\%). The accessibility of related information predicted TOT rates, which is supportive of the views of Koriat (1993, 1995). The conclusion is that the retrieval of related information powers TOTs, rather than the strength of the target name itself.

The retrieval of emotional information has also been shown to affect the likelihood of a TOT: A cue that arouses emotion is seen as more likely to be retrievable than a cue that does not arouse emotion. Schwartz (2010) examined TOTs for two kinds of general-information questions: questions that aroused emotional responses (e.g., "What is the term for ritual suicide in Japan?") and those that did not arouse emotions (e.g., "What is the capital of Denmark?"). After unsuccessful recall, there was no difference between emotional and nonemotional questions in the ability of participants to correctly recognize the correct answer. Therefore, the two sets of questions were equated in terms of memory strength, and the direct-access model therefore predicts that there should have been no differences in the rates of TOTs for the two classes of items. However, the heuristic-metacognitive model allows for the possibility that the experience of emotion could inform the experience of a TOT. The results showed that TOTs increased for the emotional items relative the nonemotional items, again supporting the heuristic-metacognitive model.

The retrieval of information concerning the past success or retrieval of the inaccessible target likely also influences TOTs. Warriner and Humphreys (2008) examined TOTs at two points, during an initial test and then a second test $48 \mathrm{~h}$ later. Their questions were whether or not participants would experience TOTs for the same or for different items, and whether being stuck in a TOT for longer, initially, would increase the likelihood of being stuck again. In the initial test, once a TOT had occurred, the experimenter manipulated the attempted retrieval interval such that some participants spent $10 \mathrm{~s}$ trying to resolve their TOTs, while others spent $30 \mathrm{~s}$ trying to resolve them. After the 10- or the 30-s delay, participants were shown the correct answer. Warriner and Humphreys then gave a second test to participants $48 \mathrm{~h}$ later. In this second test, the participants had equal amounts of time to attempt retrieval. Participants who had spent more time trying to recover the TOT targets initially were more likely to experience a TOT to these queries again than were those who had spent less time trying to recover the TOT initially. Although Warriner and Humphreys's interests were elsewhere, our interpretation of these results is that even when participants have forgotten the semantic information related to the target answer, they may have remembered the event of being in the TOT itself, and this remembrance of the initial TOT state may have contributed to their feeling of being in a TOT state again. That is, remembering that one was in a TOT state may serve as relevant related information that may drive a TOT the next time around. Thus, remembering past retrieval failures may serve as another clue that informs the inferential process of TOTs.

Finally, Cleary, Konkel, Nomi, and McCabe (2010) examined TOTs for the names of presented and unpresented odors. At the time of test, participants were presented with scents and asked to generate the names that described the odors. If they could not recall the name, they were asked if they were in a TOT for the name of that odor and if the odor had been presented in an earlier phase of the study. The authors found that being in a TOT predicted that a participant would indicate that the odor had been smelled before, regardless of whether it had been nor not. That is, if a TOT was indicated concerning an odor, an attribution was made that the odor had been studied. Here, too, TOTs were related to attributional processes rather than direct access to the name of the smell. Thus, the data from the Cleary et al. 
(2010) study are also consistent with the heuristicmetacognitive account.

It is noteworthy that in the Cleary et al. (2010) study, as in others, the monitoring system may produce a spurious TOT if there is sufficient heuristic information but the actual target is not represented in memory. Under normal circumstances, however, TOTs accurately predict eventual recall as well as recognition (Brown, 2011). Nonetheless, because the experience results from this heuristic process, researchers can induce TOT experiences by increasing the familiarity of the cue or providing nondiagnostic related information.

Neuroimaging evidence for the heuristic-metacognitive account

Direct-access models suggest that the areas of the brain active in TOTs might be restricted to the same areas that are involved in the act of retrieval. In contrast, the heuristic-metacognitive account suggests that TOTs arise not only from retrieval, but also from monitoring functions. Two studies using fMRI are supportive of the heuristic-metacognitive account. These studies were designed to examine the functional regions involved in TOTs, and both point to areas of the prefrontal lobe as being implicated. Maril, Wagner, and Schacter (2001) and Maril, Simons, Weaver, and Schacter (2005), in similar studies, presented definitions of words and generalinformation questions and asked participants to retrieve the word that matched them. For example, in Maril et al. (2005), the participants were given a cue such as "Carmen, composer" and were expected to generate the target (e.g., "Bizet"). Participants made one of three responses, indicating that (1) they were recalling the answer, (2) they did not know the answer, or (3) they were in a TOT for the target. The authors then compared the brain activity across these three conditions. Retrieval areas of the brain were activated during all conditions, but activation was strongest during successful retrieval. The study found that the areas of the brain uniquely activated during TOTs were mostly in the right prefrontal lobe. These areas included the anterior cingulate, the right dorsolateral cortex, and the right inferior prefrontal cortex. These areas of the right prefrontal cortex are associated with a number of monitoring and supervisory functions, including executive control. The dorsolateral prefrontal cortex, for example, is associated with ignoring irrelevant perceptual details, reordering lists, and a host of other features associated with monitoring ongoing cognition (see Botvinick, 2007; Shimamura, 2008). Thus, the neuroimaging studies are consistent with the heuristic-metacognitive view of TOTs.

TOTs and the control of retrieval

To return to the tire pressure metaphor, just as the "low pressure" signal alerts us to a tire low on air, the TOT alerts us to the possibility that we might be able to retrieve a particular item with more effort. Control means the decisions we make are based on metacognitive monitoring. In the car metaphor, we can make a decision to bring the car to a gas station and put air in the tires based on the signal that we see on the dashboard. When our metacognitive systems produce a TOT, we can engage in number of strategies that may be useful for facilitating retrieval. Because we know that TOTs are usually accurate indicators that an item currently not retrieved may be retrieved soon, this system is functionally adaptive in helping us retrieve difficult-to-access targets on which we might otherwise give up. This suggests that TOTs might have a cognitive function: They provide us with information into an otherwise opaque retrieval system and signal that further effort might be warranted. Thus, it is possible to think of a TOT not as a marker of failed retrieval, but as a premonition of future recall. Of course, the empirical question remains: Do TOTs affect our retrieval behavior?

A number of studies have demonstrated a relation between TOTs and the control of retrieval. For example, Schwartz (2001) found that participants spent more time trying to resolve an unrecalled target if a TOT had occurred than if no TOT had occurred. In the study, participants attempted to retrieve the answers to general-information questions. If they failed to do so, they were asked if they experienced a TOT. More time was spent trying to retrieve items that then received TOTs than was spent trying to retrieve "don't know" responses. This study is correlational-from the results, it is possible to interpret that longer retrieval times are used as a cue to TOTs, but also possible to interpret that the TOT experience itself drives the longer retrieval attempts.

Ryan, Petty, and Wenzlaff (1982) asked participants to retrieve word definitions. If they failed to retrieve the word, they were asked if they were in a TOT. Ryan et al. had their participants engage in a concurrent number probe task. The participants were presented with a series of numbers and were required to indicate every time they saw a particular number. Performance on the task was measured by accuracy. When participants were experiencing a TOT for the word definition, they made more errors on the number probe task. Similarly, Schwartz (2008) examined dual-task performance. Participants were required to maintain a digit span while they did a TOT-prospecting task. Schwartz found that working memory performance decreased during TOTs relative to during no TOTs. These two studies suggest that participants were still allocating attention and resources to resolving TOTs, allowing performance on the secondary task to suffer. The studies also suggest that TOTs alter the way in which we attempt retrieval. Thus, TOTs alert us to temporary inaccessibility, which allows us to devote more resources to resolving the TOTs.

To summarize, we have presented data that support the notion that TOTs are caused by a heuristic-metacognitive 
mechanism. In this view, a metacognitive monitor produces TOT states based on a wide variety of information that may be available, including fragments of the target, cue-based information, the history of previous recalls, and emotional information. To support the view that TOTs are not exclusively indicative of partial target retrieval, we have described several dissociations in which TOTs occur as a function of information not related to target retrieval. In addition, the neuroimaging data are consistent with this monitoring account.

\section{Trajectory}

Much is understood about the nature of the state of semantic access, in which, despite retrieval of target information, the phonology of an item cannot be produced (Gollan \& Brown, 2006). Much is understood about the nature of the TOT state itself (Brown, 2011; Schwartz, 2006). What remains to be done is to form a synthesis of the two views. The future growth in understanding TOTs will require an understanding of both the retrieval process or processes that underlie TOTs and the metacognitive processes that give rise to this feeling state.

\section{A general model of TOTs: a synthesis of two theories}

Generally, direct-access and heuristic-metacognitive theories have been seen as competing or, at best, as not mutually exclusive (Schwartz, 2002). Direct-access theories postulate that the same events trigger retrieval failure and TOTs, whereas heuristic-metacognitive theories have seldom directly addressed what causes retrieval failure. We propose that models such as that of Gollan and Brown (2006) speak to aspects of the processes underlying people's inability to quickly and efficiently retrieve a desired target item. However, a retrieval failure alone is not sufficient for a TOT to occur; it must also result in the experience of a TOT, generated by separate processes. The TOT experience is best accounted for by the heuristicmetacognitive account. In the model, a cue is given (e.g., "What country uses the rupee as its currency?"). This starts a retrieval process. Many items will be retrieved quickly. Furthermore, many items may fail to be retrieved, but if this failure is quick and sure, the failure of retrieval will not trigger a TOT. Only when retrieval takes a considerable amount of time and still comes up short will the monitoring system be engaged, which will produce a TOT if sufficient related and cue information is accessible. The heuristicmetacognition model now comes into play, and the theory produces a set of hypotheses that account for whether or not a TOT will accompany the retrieval failure (see Fig. 1). As the person tries to retrieve, he or she brings to mind partial information relating to the question, stemming from any and all of the sources mentioned above. Given a sufficient accumulation of these fragments - that is, enough information-a TOT will be triggered. The TOT is metacognitive, insofar as it is conscious and reflects the underlying cognitive process by giving a marker of a certain amount of information accumulation. The TOT also has motivational consequences of its own: It indicates to the individual that access of the target might still be possible, and that he or she should continue to try to accumulate information.

This view opens the possibility that the relation between slow and laborious retrieval and a potential taxonomy of TOTs may be more complex than previously considered. For example, consider the question "What country uses the rupee as its currency?" We conjecture that retrieval could fail for two reasons. First, the cue may be insufficiently linked to the target to fully activate it, or second, the target itself may have insufficient activation to be recalled quickly. That is, retrieval may fail because of weakened associations between the cue and target, or because the target itself is not represented strongly enough to promote recall, regardless of the cue. In the first case, the association between rupee and India is not strong, but given another cue (e.g., "What country was Mahatma Gandhi from?"), "India" may be activated. In the second case, "India" will be unlikely to be retrieved regardless of the cue because of insufficient activation of the word itself. Our question here is whether each type of retrieval failure elicits the same kind of TOT or whether there are separate subjective experiences associated with each type of retrieval failure.

\section{Unresolved issues}

Other issues related to TOTs have not yet been fully explored. These areas remain important to investigate because they both bear on the theoretical issues and may have practical applications. We consider several of these issues for which some research exists, but more needs doing.

Tip-of-the-finger, tip-of-the-pen, and tip-of-the nose all refer to phenomena closely related to the TOT. ASL users have an expression that translates as "tip-of-the-finger," in which a person is sure that he or she knows the visual sign for the concept but cannot recall the movements of the hand that express the meaning (Thompson et al., 2005). Research suggests some similarities but also some differences between TOTs and tip-of-the finger experiences (Thompson et al., 2005). Chinese readers have described a "tip-of-thepen" phenomenon, in which a Chinese speaker knows the spoken word and feels as if he or she is about to recall how to make the unrecalled written character for that spoken word (Sun, Vinson, \& Vigliocco, 1998). Except for the one study just referenced, there has been no work on this phenomenon. The "tip-of-the-nose" phenomenon refers to the strong experience that the name of a familiar odor will 
be recalled in the presence of the particular odor (see Cleary et al., 2010). Jonsson and Olssen (2003) found that tip-ofthe-nose experiences were associated with later recall and recognition but that they were not associated with more partial information than were unretrieved odor names that did not elicit tip-of-the-nose experiences. It is likely that the processes for all three of the phenomena here are similar to those for TOTs, but more research is required.

Another unresolved issue is whether or not the TOT is a single phenomenon or a group of related experiences. It is possible that the TOT is a discrete experience that occurs in an "all-or-nothing" manner when particular circumstances are met. This is typically the manner in which the TOT is treated in the literature. Most studies ask whether participants are in a TOT or not for unrecalled items. However, it is possible that the TOT can be treated, like other metacognitive judgments, in a graded fashion, varying from strong to weak (see Brown, 2011). Indeed, people are able to distinguish between what they call "blocked" and "pure" TOTs, and there are some empirical differences that correspond to that distinction. Several other studies have examined distinctions among TOTs (e.g., Gardiner, Craik, \& Bleasdale, 1973; Kozlowski, 1977; Schwartz et al., 2000), showing that people can make distinctions among other TOT substates that correlate with some aspects of behavior. Schwartz et al. (2000), for example, found that stronger, more emotional, and more "imminent" TOTs were more likely to be followed by resolution and recognition than were those judged less strong, less emotional, or less imminent. Similarly, Gardiner et al. found that recognition rates increased with the numerical evaluation of the strength of a TOT rating.

Another topic that merits more consideration is the relation between TOTs and emotion. Anecdotally, many reports link TOTs to emotion (e.g., James, 1890). However, the exact interplay between TOTs and emotion has not been specified. We suspect that emotional responses are a source of information that is used in the calculus of the TOT production system. That is, if a cue or question evokes emotion, that emotional information may increase the likelihood of a TOT occurring. In fact, Schwartz (2010) found that emotioninducing questions led to more TOTs than did neutral questions. On the other hand, it may be that retrieval failure is frustrating, and knowledge of that retrieval failure is even more frustrating. Indeed, Brown and McNeill (1966) described the experience of being in a TOT as one of being in "mild torment" (p.326). In this case, it may be the TOT that has already occurred that drives the experience of emotion.

\section{Closure}

TOTs allow us to explore the nature of word retrieval and the workings of retrieval failure, as well as providing a case study in phenomenology. Thus, TOTs offer great value in terms of their theoretical interest. The study of TOTs has practical value as well, since understanding them may be useful in designing learning tools, helping older adults overcome memory problems, and diagnosing neurological illnesses. We consider each of these possibilities in turn.

Learning aids

People typically think of TOTs as a problem - that is, a failure to recall a known word. However, when viewed from the metacognitive perspective, a TOT can be considered functionally adaptive (see Gollan \& Brown, 2006): If we experience a TOT, we spend more time trying to retrieve the item. Because TOTs are correlated with the ability to overcome retrieval failure, attending to our own TOTs is adaptive. In addition, studies show that when a person spends more time retrieving a difficult item, that item will be better learned (Benjamin, Bjork, \& Schwartz, 1998). Thus, TOTs may lead to stronger encoding of the item than do retrieval failures not accompanied by a TOT (but see Warriner \& Humphreys, 2008). We suggest that a successful research project in the future would be to create conditions that produce differential rates of TOTs for similarly difficult items.

\section{Dyslexia}

Research shows that TOTs are more common among dyslexic children than among normal control children (Hanly \& Vandenberg, 2010). Training dyslexic children in methods of overcoming TOTs (such as first-letter cuing; see Schwartz, 2002) may help them learn to access semantic information faster and to process phonological information more quickly, and therefore to read better. This may involve encouraging the child to retrieve a known word rather than having a parent or teacher provide it to them. In this way, the child can benefit from the experience of retrieving him- or herself. Many recent data support the idea that retrieval can act as a potent learning tool (Kornell \& Son, 2009; Roediger \& Karpicke, 2006).

Brain pathologies

Anomic aphasia is defined as an acquired deficit in word retrieval (Laine \& Martin, 2006). Research has shown that anomic aphasics experience many TOTs as well (Funnell, Metcalfe, \& Tsapkini, 1996). This understudied area could tell us much about the nature of anomia, as well as providing much information about TOTs, since the anomic patient described by Funnell et al. was in a virtually constant state of TOTs. 
Older adults

Older adults experience more TOTs than younger adults (see Brown, 2011, for a review). Some researchers account for this effect by asserting that older adults are more likely to show retrieval failure of known words (e.g., Heine et al., 1999). Thus, increased TOTs in older age have typically been seen as a negative development. However, from a metacognitive perspective, the experience of a TOT may be a good thing. It informs the older adult that the knowledge is present, even if he or she is currently unable to access it (Schwartz \& Frazier, 2005). If older adults were taught the metacognitive perspective, they might view their TOTs as less frustrating and as an indication that more "search" time will eventually generate the target. However, to date, there has been no empirical research on TOTs and aging motivated by the heuristic-metacognitive approach.

\section{Judgment}

TOTs continue to interest researchers in metacognition (e.g., Schwartz, 2010), memory retrieval (e.g., Kornell \& Metcalfe, 2006), and language production (e.g., Biedermann, Ruh, Nickels, \& Coltheart, 2008; Warriner \& Humphreys, 2008). The extension of the study of TOTs may be beneficial to people who are aging or have difficulties with memory retrieval or lexical access. Each of these areas promises to contribute to a growing understanding of the TOTs themselves and of the role that this phenomenological state plays in human cognition. TOTs are conscious experiences that exist across cultures, languages, and ages (Brennen et al., 2007; Brown \& Nix, 1996). Since Brown and McNeill's (1966) study, we have been able to bring TOTs into the lab and study them under controlled experimental conditions. Under these conditions, it is possible to explain what TOTs are to participants and to generate them using the prospecting procedure.

There are still unresolved issues that await further research. But progress has been made in coming to a more firm understanding of the underlying mechanisms that contribute to TOTs and to their function. TOTs continue to offer us insights into the nature of cognition - in terms of both the mechanisms of retrieval failure and the metacognitive mechanisms that monitor retrieval and motivate renewed efforts. The study of TOTs provides fertile ground for research on the relation of metacognition and the control of behavior. Our synthesis model describes an accumulative retrieval process in which the content of the information retrieved may vary across a variety of domains, but in which a sufficient amount of information triggers the TOT state. This model is not isomorphic to the two-level "directaccess" view of TOTs (Gollan \& Brown, 2006). Neverthe- less, it provides a framework that can encompasses the twolevel view, which accounts for retrieval failure, and metacognitive views, which account for the phenomenology. This new synthesis was designed to accommodate both the psycholinguistic and the metacognitive data. More importantly, it highlights the function of TOTs in guiding further memory retrieval and lexical access processes. Namely, we propose that the function of TOTs is to alert us to the possibility that the apparent current retrieval failure in fact has the potential for future retrieval success, and therefore to direct us to appropriate continued retrieval efforts. This focus on the function of TOTs may be fruitful in applying the work on TOT states to conditions such as aging or dyslexia or anomia, as well as in more accurately describing the role of TOTs in normal human cognition.

\section{References}

Bacon, E., Schwartz, B. L., Paire-Ficout, L., \& Izaute, M. (2007). Dissociation between the cognitive process and the phenomenological experience of the TOT: Effect of the anxiolytic drug lorazepam on TOT states. Cognition and Consciousness, 16, $360-373$.

Benjamin, A. S., Bjork, R. A., \& Schwartz, B. L. (1998). The mismeasure of memory: When retrieval fluency is misleading as a metamnemonic index. Journal of Experimental Psychology: General, 127, 55-68.

Biedermann, B., Ruh, N., Nickels, L., \& Coltheart, M. (2008). Information retrieval in tip of the tongue states: New data and methodological advances. Journal of Psycholinguistic Research, 37, 171-198.

Botvinick, M. (2007). Conflict monitoring and decision making: Reconciling two perspectives on anterior cingulate function. Cognitive, Affective \& Behavioral Neuroscience, 7, 356-366.

Brennen, T., Vikan, R., \& Dybdahl, R. (2007). Are tip-of-the-tongue states universal? Evidence from an unwritten language. Memory, 15, 167-176. doi:10.1080/09658210601164743

Brown, A. S. (1991). A review of the tip-of-the-tongue experience. Psychological Bulletin, 109, 204-223. doi:10.1037/00332909.109.2.204

Brown, A. S. (2003). A review of the déjà vu experience. Psychological Bulletin, 129, 394-413.

Brown, A. S. (2004). The déjà vu illusion. Current Directions in Psychological Science, 13, 256-259.

Brown, A. S. (2011). Tip of the tongue state. Hove, U.K.: Psychology Press.

Brown, R., \& McNeill, D. (1966). The "tip of the tongue" phenomenon. Journal of Verbal Learning and Verbal Behavior, 5, 325-337. doi:10.1016/S0022-5371(66)80040-3

Brown, A. S., \& Nix, L. A. (1996). Age differences in the tip-of-thetongue experience. The American Journal of Psychology, 109, $79-91$.

Cleary, A. M. (2006). Relating familiarity-based recognition and the tip-of-the-tongue phenomenon: Detecting a word's recency in the absence of access to the word. Memory \& Cognition, 34, 804816.

Cleary, A. M., Konkel, K. E., Nomi, J. S., \& McCabe, D. P. (2010). Odor recognition without identification. Memory \& Cognition, $38,452-460$. 
Cleary, A. M., Ryals, A. J., \& Nomi, J. N. (2009). Can déjà vu result from similarity to a prior experience? Support for the similarity hypothesis of déjà vu. Psychonomic Bulletin \& Review, 16, $1082-1088$.

Costall, A. (2006). "Introspectionism" and the mythical origins of scientific psychology. Consciousness and Cognition, 15, 634-654.

Dahlgren, D. J. (1998). Impact of knowledge and age on tip-of-thetongue rates. Experimental Aging Research, 24, 139-153.

Dunlosky, J., \& Metcalfe, J. (2009). Metacognition. Thousand Oaks, CA: Sage.

Funnell, M., Metcalfe, J., \& Tsapkini, K. (1996). In the mind but not in the tongue: Feeling of knowing in anomic patient H.W. In L. M. Reder (Ed.), Implicit memory and metacognition (pp. 171-194). Hillsdale, NJ: Erlbaum.

Galdo-Alvarez, S., Lindin, M., \& Diaz, F. (2009). The effect of age on event-related potentials (ERP) associated with face naming and the tip-of-the-tongue (TOT) state. Biological Psychology, 81, 1423. doi:10.1016/j.biopsycho.2009.01.002

Gardiner, J. M., Craik, F. I. M., \& Bleasdale, F. A. (1973). Retrieval difficulty and subsequent recall. Memory \& Cognition, 1, 213-216.

Gollan, T. H., \& Acenas, L. A. (2004). What is a TOT? Cognate and translation effects on tip-of-the-tongue states in SpanishEnglish and Tagalog-English bilinguals. Journal of Experimental Psychology. Learning, Memory, and Cognition, 30, 246-269.

Gollan, T. H., \& Brown, A. S. (2006). From tip-of-the-tongue (TOT) data to theoretical implications in two steps: When more TOTs means better retrieval. Journal of Experimental Psychology: General, 135, 462-483. doi:10.1037/0096-3445.135.3.462

Hamberger, M. J., \& Seidel, W. T. (2003). Auditory and visual naming tests: Normative and patient data for accuracy, response time, and tip-of-the-tongue. Journal of the International Neuropsychological Society, 9, 479-489.

Hanley, J. R., \& Chapman, E. (2008). Partial knowledge in a tip of the tongue state about two and three word proper names. Psychonomic Bulletin \& Review, 15, 156-160.

Hanly, S., \& Vandenberg, B. (2010). Tip-of-the-tongue and word retrieval deficits in dyslexia. Journal of Learning Disabilities, 43, 15-23.

Harley, T. A., \& Bown, H. E. (1998). What causes a tip-of-the-tongue state? Evidence for lexical neighbourhood effects in speech production. British Journal of Psychology, 89, 151-174.

Hazy, T. E., Frank, M. J., \& O'Reilly, R. C. (2007). Toward an executive without a homunculus: Computational models of the prefrontal cortex/basal ganglia system. Philosophical Transactions of the Royal Society B, 362, 1601-1613.

Heine, M. K., Ober, B. A., \& Shenaut, G. K. (1999). Naturally occurring and experimentally induced tip-of-the-tongue experiences in three adult age groups. Psychology and Aging, 14, $445-457$

James, W. (1890). The principles of psychology. New York: Holt (Original work published 1864).

Jonsson, F. U., \& Olssen, M. A. (2003). Olfactory metacognition. Chemical Senses, 28, 651-658. doi:10.1093/chemse/bjg058

Kelly, R. (2009). The will of Achilles. Available from www. conjunctions.com/webcon/kelly-209.htm

Koriat, A. (1993). How doe we know that we know? The accessibility account of the feeling of knowing. Psychological Review, 100, 609-639.

Koriat, A. (1995). Dissociating knowing and the feeling of knowing: Further evidence for the accessibility model. Journal of Experimental Psychology: General, 124, 311-333.

Koriat, A., \& Lieblich, I. (1974). What does a person in a "TOT" state know that a person in a "don't know" state doesn't know. Memory \& Cognition, 2, 647-655.

Koriat, A., \& Lieblich, I. (1977). A study of memory pointers. Acta Psychologica, 41, 151-164.
Kornell, N., \& Metcalfe, J. (2006). "Blockers" do not block recall during tip-of-the-tongue states. Metacognition and Learning, 1, 248-261. doi:10.1007/s11409-007-9003-z

Kornell, N., \& Son, L. K. (2009). Learners' choices and beliefs about self-testing. Memory, 17, 493-501.

Kozlowski, L. T. (1977). Effects of distorted auditory and of rhyming cues on retrieval of tip-of-the-tongue words by poets and nonpoets. Memory \& Cognition, 5, 477-481.

Laine, M., \& Martin, N. (2006). Anomia: Theoretical and clinical aspects. New York: Psychology Press.

Lesk, V. E., \& Womble, S. P. (2004). Caffeine, priming and tip of the tongue: Evidence for plasticity in the phonological system. Behavioral Neuroscience, 118, 453-461.

Maril, A., Simons, J. S., Weaver, J. J., \& Schacter, D. L. (2005). Graded recall success: An event-related fMRI comparison of tip of the tongue and feeling of knowing. Neuroimage, 24, 1130-1138.

Maril, A., Wagner, A. D., \& Schacter, D. L. (2001). On the tip of the tongue: An event-related fMRI study of semantic retrieval failure and cognitive conflict. Neuron, 31, 653-660.

Metcalfe, J. (1993). Novelty monitoring, metacognition, and control in a composite holographic associative recall model: Interpretations for Korsakoff amnesia. Psychological Review, 100, 3-22.

Metcalfe, J., Schwartz, B. L., \& Joaquim, S. G. (1993). The cue familiarity heuristic in metacognition. Journal of Experimental Psychology. Learning, Memory, and Cognition, 19, 851-861. doi:10.1037/0278-7393.19.4.851

Miozzo, M., \& Caramazza, A. (1997). Retrieval of lexical-syntactic features in tip-of-the-tongue states. Journal of Experimental Psychology. Learning, Memory, and Cognition, 23, 1410-1423.

Perfect, T. J., \& Hanley, J. R. (1992). The tip-of-the-tongue phenomenon: Do experimenter-presented interlopers have any effect? Cognition, 45, 55-75.

Roediger, H. L., III, \& Karpicke, J. D. (2006). Test-enhanced learning: Taking memory tests improves long-term retention. Psychological Science, 17, 249-255.

Ryan, M. P., Petty, C. R., \& Wenzlaff, R. M. (1982). Motivated remembering efforts during tip-of-the-tongue states. Acta Psychologica, 51, 137-147.

Schacter, D. L. (2001). The seven sins of memory: How the mind forgets and remembers. Boston: Houghton Mifflin.

Schwartz, B. L. (1999). Sparkling at the end of the tongue: The etiology of tip-of-the-tongue phenomenology. Psychonomic Bulletin \& Review, 6, 379-393.

Schwartz, B. L. (2001). The relation of tip-of-the-tongue states and retrieval time. Memory \& Cognition, 29, 117-126.

Schwartz, B. L. (2002). Tip-of-the-tongue states: Phenomenology, mechanism, and lexical retrieval. Mahwah, NJ: Erlbaum.

Schwartz, B. L. (2006). Tip-of-the-tongue states as metacognition. Metacognition and Learning, 1, 149-158.

Schwartz, B. L. (2008). Working memory load differentially affects tip-of-the-tongue states and feeling-of-knowing judgment. Memory \& Cognition, 36, 9-19. doi:10.3758/MC.36.1.9

Schwartz, B. L. (2010). The effects of emotion on tip-of-the-tongue states. Psychonomic Bulletin \& Review, 17, 82-87.

Schwartz, B. L., \& Frazier, L. D. (2005). Tip-of-the-tongue states and aging: Contrasting psycholinguistic and metacognitive perspectives. The Journal of General Psychology, 132, 377-391.

Schwartz, B. L., \& Smith, S. M. (1997). The retrieval of related information influences tip-of-the tongue states. Journal of Memory and Language, 36, 68-86. doi:10.1006/jmla.1996.2471

Schwartz, B. L., Travis, D. M., Castro, A. M., \& Smith, S. M. (2000). The phenomenology of real and illusory tip-of-the-tongue states. Memory \& Cognition, 28, 18-27.

Sedikides, C., Wildschut, T., Arndt, J., \& Routledge, C. (2008). Nostalgia: Past, present, and future. Current Directions in Psychological Science, 17, 304-307. 
Shimamura, A. P. (2008). A neurocognitive approach to metacognitive monitoring and control. In J. Dunlosky \& R. A. Bjork (Eds.), Handbook of memory and metamemory: Essays in honor of Thomas O. Nelson (pp. 373-390). New York: Psychology Press.

Simner, J., \& Ward, J. (2006). The taste of words on the tip of the tongue. Nature, 444, 438-438.

Smith, S. M. (1994). Frustrated feelings of imminent recall: On the tip-of-the tongue. In J. Metcalfe \& A. P. Shimamura (Eds.), Metacognition: Knowing about knowing (pp. 27-46). Cambridge, MA: MIT Press.

Sun, Y., Vinson, D. P., \& Vigliocco, G. (1998). Tip-of-the-tongue and tipof-the-pen in Chinese. Abstracts of the Psychonomic Society, 3, 32.

Thompson, R., Emmorey, K., \& Gollan, T. (2005). Tip-of-the-fingers experiences by ASL signers: Insights into the organization of a sign-based lexicon. Psychological Science, 16, 856-860.
Tulving, E. (1989). Memory: Performance, knowledge, and experience. European Journal of Cognitive Psychology, 1, 3-26.

Warriner, A. B., \& Humphreys, K. R. (2008). Learning to fail: Reoccurring tip-of-the-tongue states. The Quarterly Journal of Experimental Psychology, 61, 535-542.

Woodworth, R. S. (1931). Contemporary schools of psychology. London: Methuen.

This research was supported by National Institute of Mental Health Grant RO1-MH60637; Grant R305H060161 from the Institute of Educational Sciences, Department of Education; and Grant 220020166 from the James S. McDonnell Foundation. The authors are wholly responsible for the content of this article, and thank Moses Aluicio and Leslie Frazier for comments on drafts of the manuscript. 